\title{
Assessment of Autonomic Nerve Function In Hypothyroids By Time Domain Method of Heart Rate Variability
}

\author{
Matia Ahmed ${ }^{1}$, Noorzahan Begum ${ }^{2}$, Sultana Ferdousi ${ }^{3}$
}

\begin{abstract}
Background: Cardiovascular complications are common in hypothyroids .Time domain measures of HRV is useful to determine the cardiac autonomic regulation by assessing sympathovagal balance. Objective: To assess the cardiac autonomic nerve function activity by time domain measures of heart rate variability in patients with hypothyroidism. Methods: This cross sectional study was carried out in the Department of Physiology, Banghabandhu Sheikh Mujib Medical University between July 2008 to June 2009 on 60 female patients with hypothyroidism aged 30-50 years. 30 age matched apparently healthy euthyroid subjects were also studied as control. Hypothyroid patients were divided into 2 subgroups ,one group includes patients on their 1st day of diagnosis, before starting of treatment and another group includes patients with medication for 12-18 months. The patients were selected from the Out Patients Department of Endocrinology Wing BSMMU. Serum TSH and FT4 levels of all subjects were measured by AxSYM system. For assessing HRV by time domain method, mean R-R interval, mean heart rate, SDNN, RMSSD and variance were measured by a Polyrite. For statistical analysis, Independent Sample t test and One Way ANOVA were used. Results: The mean serum TSH was significantly higher and $\mathrm{FT}_{4}$ was significantly lower $(\mathrm{p}<0.001)$ in newly diagnosed hypothyroids than those of control and treated group. In untreated hypothyroid patients the mean values of heart rate, SDNN, RMSSD, variance were found significantly $(\mathrm{p}<0.001)$ lower in comparison to both control and treated patients. No significant difference in these hormonal levels and all these time domain measures were found between control and treated group. Conclusion: This study concludes that decrease parasympathetic nerve activity may be a consequence of hypothyroidism, if not treated properly.
\end{abstract}

Keywords: HRV, mean heart rate, SDNN, RMSSD, Hypothyroidism.

J Bangladesh Soc Physiol. 2012 June; 7(1): 48-52 For Authors Affiliation, see end of text.

http://www.banglajol.info/index.php/JBSP

\section{Introduction}

7 he thyroid gland produces two major hormones, thyroxine and triiodothyronine. This hormone affects every organ and organ system. Hypothyroidism may be associated with different cardiovascular and metabolic disorders including autonomic

Received Feb 14 2012; $\quad$ Accepted June 2012 nerve dysfunction. Many researchers reported the relationship between hypothyroidism and changes in cardiac autonomic nerve activities (CANA). They observed increased sympathetic and decreased parasympathetic nerve activity in these group hypothyroid patients. ${ }^{1-2}$ Different investigators, studied time domain parameters in hypothyroid patients to observe their autonomic nerve function status. In their study most of them 
had shown that deficiency of thyroid hormone caused lower heart rate in hypothyroids. ${ }^{1-3}$ In addition several studies reported lower values of SDNN ,RMSSD and variance in untreated hypothyroids than those of euthyroids ${ }^{4-6}$. Moreover some researchers suggested that thyroid releasing hormone stimulates sympathetic outflow in CNS thereby causing its increased activity in hypothyroids.

In our country several studies on autonomic nerve function status was done in some clinical condition such as hypertension, hyperthyroidism, and also in health like sedentary worker and athelets ${ }^{7-10}$ but no published data is yet available based on relation of thyroid hormone deficiency with autonomic nerve activity by time domain method. Therefore, the present study has been designed to assess the autonomic nerve function status in hypothyroids by measuring time domain parameters of HRV. This would raise consciousness among the clinicians and patients that early of treatment can minimize hypothyroid related cardiovascular complications.

\section{Methods}

This cross sectional study was carried out to observe the autonomic nerve function by time domain method of HRV in 60 hypothyroid female patients (group B) aged 30-50 years in the department of Physiology, Bangabandhu Sheik Mujib Medical University between July 2008 to June 2009. For comparison 30 age and sex matched apparently healthy euthyroid subjects were also studied. Hypothyroid patients were further subdivided according to treatment given into newly diagnosed untreated case and under treatment for about 2 years. The hypothyroid cases were selected from the Out Patient Department of the Endocrinology Wing of the department of medicine, BSMMU, and the controls were selected from the same area by personal contact. All the subjects were free from heart disease, hypertension, diabetes mellitus, chronic renal failure, psychic disorders and smoking. After selection, the subject was thoroughly informed about the aim, objectives and detail procedure before commencing the study and collection of blood sample. They were encouraged for voluntary participation. If she agreed to enroll to the study, informed written consent was taken from them. To prepare autonomic nerve function test the subjects were advised to have their meal by 9:00 pm on previous night, to remain free from any physical or mental stress, not to take any drugs affecting central nervous system and to have a good sleep at night before the examination day. The subject were advised to avoid tea or coffee at breakfast and to attend the Autonomic Nerve Function Test Laboratory between 9:00 to 11:00 a.m. on the day of study, the subject were interviewed and detail history regarding personal history, drug history, past medical history was taken. Then thorough physical examinations and anthropometric parameters like height, weight, BMI, were taken and the information was recorded in a prefixed questionnaire. Then the subjects were kept under complete bed rest in supine position for 20 minutes in a cool and calm environment. During this period she was advised not to talk, eat or drink and also not to perform physical or any mental activity, even sleep. Then all preparations for recording of the Heart Rate Variability parameters were made by connecting the channels of ECG to a polygraph and 5 minutes recording was taken in resting supine position. Time domain measures of Heart rate variability like mean R-R interval, mean heart rate, SDNN and RMSSD and variance were recorded. After recording is over $5 \mathrm{ml}$ of venous blood was drawn from the subjects. The serum level of thyroid hormone was measured by Micro particle enzyme immuno assay (MEIA) method. Data were expressed as mean $\pm \mathrm{SD}$. For statistical analysis Independent Sample t test and one way ANOVA were used as applicable. 


\section{Result}

All groups were matched for age and BMI. The mean serum TSH was significantly higher $(\mathrm{p}<0.001)$ and $\mathrm{FT}_{4}$ was significantly lower $(p<0.001)$ in untreated hypothyroids than those of control and treated group but no statistically significant differences were observed regarding both $\mathrm{TSH}$ and $\mathrm{FT}_{4}$ between control and treated group. (Table I). The mean values of all time domain parameters of HRV in euthyroids were within normal range. In untreated hypothyroid patients, the mean values of SDNN, RMSSD and variance were found significantly $(p<0.001)$ lower,Mean R-R interval was lower but not significant when compared with both euthyroids and treated patients. Again, no statistically significant differences were observed when all these parameters compared between euthyroids and treated group.

Table I: Serum TSH and $\mathrm{FT}_{4}$ levels in different groups $(\mathrm{n}=90)$

\begin{tabular}{lcc}
\hline Groups & TSH(mIU/L) & $\mathrm{FT}_{4}(\mathrm{pmol} / \mathrm{L})$ \\
\hline $\mathrm{A}(\mathrm{n}=30)$ & $2.18 \pm 1.04$ & $15.13 \pm 4.21$ \\
$\mathrm{~B}_{1}(\mathrm{n}=30)$ & $38.16 \pm 30.50$ & $5.12 \pm 1.89$ \\
$\mathrm{~B}_{2}(\mathrm{n}=30)$ & $2.04 \pm 1.01$ & $15.08 \pm 3.95$ \\
\hline Statistical analysis: & & \\
\hline Groups & P value & $0.000^{* * *}$ \\
\hline A vs B vs B $_{2}{ }^{\mathrm{a}}$ & $0.000^{* * *}$ & $0.000^{* * *}$ \\
$\mathrm{~A}_{\text {vs B }}{ }^{\mathrm{b}}$ & $0.000^{* * *}$ & $0.964^{\mathrm{ns}}$ \\
$\mathrm{A}^{\mathrm{n}} \mathrm{B}_{2}{ }^{\mathrm{b}}$ & $0.608^{\mathrm{ns}}$ & $0.000^{* * *}$ \\
$\mathrm{~B}_{1}$ vs B $_{2}{ }^{\mathrm{b}}$ & $0.000^{* * *}$ & \\
\hline
\end{tabular}

Table II: Simple time domain measures of HRV in different groups $(n=90)$

\begin{tabular}{|c|c|c|}
\hline Groups & Mean R-R (sec) & Mean HR(bpm) \\
\hline$A(n=30)$ & $0.75 \pm 0.8$ & $77.5 \pm 6.37$ \\
\hline $\mathrm{B}_{1}(\mathrm{n}=30)$ & $0.76 \pm 0.11$ & $71.3 \pm 4.99$ \\
\hline $\mathrm{B}_{2}(\mathrm{n}=30)$ & $0.74 \pm .09$ & $77.3 \pm 6.82$ \\
\hline \multicolumn{3}{|c|}{ Statistical analysis: } \\
\hline Groups & $P$ value & \\
\hline$A$ vs $B_{1}$ vs $B_{2}^{a}$ & $0.531^{\mathrm{ns}}$ & $0.000^{* * *}$ \\
\hline $\mathrm{A}$ vs $\mathrm{B}_{1}{ }^{\mathrm{b}}$ & $0.608^{\mathrm{ns}}$ & $0.000^{* * *}$ \\
\hline $\mathrm{A}$ vs $\mathrm{B}_{2}^{\mathrm{b}}$ & $0.567^{\mathrm{ns}}$ & $0.892^{\mathrm{ns}}$ \\
\hline $\mathrm{B}_{1}$ vs $\mathrm{B}_{2}^{\mathrm{b}}$ & $0.32^{\mathrm{ns}}$ & $0.000^{* * *}$ \\
\hline
\end{tabular}

were expressed as mean \pm SD. Statistical analysis were done by One way ANOVA ${ }^{\mathrm{a}}$ and Independent sample ' $\mathrm{t}$ ' test

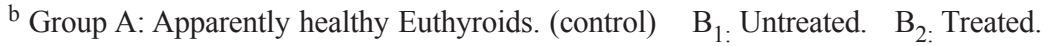

$\mathrm{TSH}=$ Thyroid stimulating hormone $\mathrm{FT}_{4}=$ Thyroxin (Free form). $\mathrm{R}-\mathrm{R}=$ Interval between successive QRS complex, HR $=$ Heart rate. $* * *=\mathrm{P}<0.001 . \mathrm{ns}=$ non significant 
Table III: Time domain measures of HRV in different groups ( $\mathrm{n}=90)$

\begin{tabular}{|c|c|c|c|}
\hline Groups & SDNN (ms) & RMSSD(ms) & Variance $\left(\mathrm{ms}^{2}\right)$ \\
\hline $\mathrm{A}(\mathrm{n}=30)$ & $64.16 \pm 18.25$ & $41.03 \pm 38.16$ & $2702 \pm 1297.89$ \\
\hline $\mathrm{B}_{1}(\mathrm{n}=30)$ & $50.16 \pm 17.43$ & $20.65 \pm 20.73$ & $1177.11 \pm 584.04$ \\
\hline$\underline{\mathrm{B}_{2}(\mathrm{n}=30)}$ & $63.99 \pm 21.87$ & $41.10 \pm 40.59$ & $2683.73 \pm 1275.87$ \\
\hline \multicolumn{4}{|c|}{ Statistical analysis: } \\
\hline Groups & & P value & \\
\hline$A$ vs $B_{1}$ vs $B_{2}^{a}$ & $0.007^{* *}$ & $0.033^{*}$ & $0.000^{* * *}$ \\
\hline $\mathrm{A}$ vs $\mathrm{B}_{1}^{\mathrm{b}}$ & $0.004^{* *}$ & $0.013^{*}$ & $0.000^{* * * *}$ \\
\hline $\mathrm{A} v \mathrm{~B}_{2}{ }^{\mathrm{b}}$ & $0.974^{\mathrm{ns}}$ & $0.995^{\mathrm{ns}}$ & $0.954^{\mathrm{ns}}$ \\
\hline $\mathrm{B}_{1} \mathrm{vs} \mathrm{B}_{2}^{\mathrm{b}}$ & $0.009^{* *}$ & $0.017^{*}$ & $0.000^{* * *}$ \\
\hline
\end{tabular}

Data were expressed as mean \pm SD. Statistical analysis were done by One way ANOVA $^{\mathrm{a}}$ and Independent sample ' $t$ ' test ${ }^{b}$. Group A: Apparently healthy Euthyroids.(control) $B_{1}$. Untreated. $B_{2}$. Treated. $\mathrm{SDNN}=$ Standard deviation of $N N$ interval, $N N$ interval= Normal to normal intervals (Interval between adjacent QRS complexes from SA node depolarization) $\mathrm{RMSSD}=$ Square root of mean squared differences of successive $N N$ intervals, ms $=$ millisecond. $\mathrm{ms}^{2}=$ millisecond square $*=\mathrm{p}<0.05 * *=\mathrm{P}<0.01 * * *=\mathrm{p}<0.001 \mathrm{~ns}=\mathrm{p}>0.05 \quad \mathrm{n}=$ number of subject

\section{Discussion}

In last decade several investigators had assessed cardiac autonomic activity by time domain measure of HRV from the ECG recording in hypothyroids patients. The present study was undertaken to observe the heart rate variability (HRV) in hypothyroid female patients in order to assess their cardiac autonomic nervous activity (CANA). In this study some time domain measures of HRV such as mean R-R interval, mean heart rate, SDNN, RMSSD,variance in untreated and treated hypothyroid cases were assessd. In this study, lower values of all time domain measures in untreated hypothyroids compared to control and treated patients indicate reduced vagal modulation. The TSH and $\mathrm{FT}_{4}$ levels in treated group has shown close to the control value which demonstrates the improvement of hormone level due to treatment. This decrease heart rate in untreated hypothyroids may be due to desensitization of receptor and also due to attenuated cardiac chronotropic response to adrenergic stimulation ${ }^{11}$. The value of SDNN was significantly lower in untreated hypothyroids in comparison to those of euthyroids and treated hypothyroid. But statistically no significant difference of this value was observed between euthyroids and treated hypothyroids. Similar significant change in SDNN values was also observed by various investigators. ${ }^{4-6}$ In this study, RMSSD was determined from a short term recording of ECG. In untreated hypothyroid patients, the RMSSD was significantly lower in comparison to those of euthyroids and treated hypothyroid. However, there was no statistically significant difference of this value was observed between euthyroids and treated hypothyroids. Similar type of finding was also reported by some investigators of other countries ${ }^{4,6}$ However, decreased values of SDNN, RMSSD may be due to reduced vagal tone in these groups of patients. 4-6 In addition all time domain parameters in treated hypothyroids patient shows marked improvement in autonomic nerve function due to effective Thyroid treatment. Various authors highlighted about the role of thyroid hormone in autonomic nerve function. They suggested that increase in sympathetic activity possibly due to thyroid releasing hormone which directly stimulates sympathetic outflow within the $\mathrm{CNS}^{8}$. 
This effect caused ruduced vagal modulation in hypothyroids due to the reciprocal inhibition between sympathetic and parasympathetic.

\section{Conclusion}

This study concludes cardiac vagal modulation may be reduced in hypothyroidism which can be improved by adequate treatment.

\section{Author affiliation :}

1. *Dr Matia Ahmed, Assistant professor, Department of Physiology,Uttara Adhunik Medical College.

2. Noorzahan Begum, Professor, Department of Physiology,BSMMU Dhaka. Email: noorzahan52 @gmail.com

3. Sultana Ferdousi, Associate Professor, Department of Physiology, BSMMU, Dhaka. Email: sferdousiratna @gmail.com

*For correspondence

\section{References}

1. Inukai T, Takanashi K, Kobayashi H, Fujiwara $\mathrm{Y}$, Tayama K, Aso Y,TakemY. Power spectral analysis of variations in heart rate in patients with Hyperthyroidism or Hypothyroidism. Horm Metab Res 1998; 30: 531-35.

2. Klein I, Ojamaa K. Thyroid hormone and the cardiovascular system. N Engl J Med 2001; 344(7): 501-509

3. Polikar R,kennedy B,Maisel A,Ziegler M, Smith J, Dittrich $\mathrm{H}$ and Nicod P. Decreased adrenergic sensitivity in patients with hypothyroidism.J Am Coll.cardiol 1990; 15: 94-98.

4. Galetta F,Frazoni F,.Fallahi P.Rossi M, Carpi.A, Rubello AntonelliA Santoro G. Heart rate variability and QT dispersion in patients with Subclinical hypothyroidism. Biomedicine\&Pharmacotherapy 2006; 425-30

5. Sahin I,Turan N,Kosar F.Evaluation of autonomic activity in patient with subclinical hypothyroidism. J Endocrinol Invest 2005; 28:209-13.

6. Xing H, Shen Y, Chen H, Wang y,Shen W. Heart rate variability and its response to thyroxine replacement therapy in patient with hypothyroidism. Clin Med J 2001; 114: 906-8.

7. Mithun S. Comparative study of cardiac autonomic nerve function status by heart rate variability between sedentary and heavy worker [Thesis] [Dhaka (Bangladesh)]: BSMMU. 2009 July.

8. Tabassum R, Begum N,Ferdousi S,Begum S,Ali T. Heart rate variability in patient with Essential Hypertension.J Bangladesh soc Physiol.2010; $5(1): 1-7$

9. Kabir MR, Begum N,Ferdousi S,Begum S,Ali T. Heart Rate Variability In Hyperthyroidism .J Bangladesh soc Physiol.2009;4(2):51-7

10. Alom M, Begum N,Ferdousi S,Begum S,Ali T. Power spectral analysis of heart rate variability in adolescent male athletes. J Bangladesh soc Physiol 2009; 4(2):26-33.

11. Cacciatori V,Gemma ML,Bellevare F. Power spectral analysis of heart rate in hypothyroidism. Eur J Endocrinol 2000; 143:327-33. 